\title{
Prediction Model of Power Consumption for Variable Material Removal Rate Machining Process
}

\author{
Yasuo Kondo, Yuko Itoh, Mitsugu Yamaguchi, Satoshi Sakamoto, and Kenji Yamaguchi
}

\begin{abstract}
We tried to examine the power consumption for material-cutting in the variable-material removal rate (MRR) machining process. In the case of constant-MRR process, the power consumption is almost in proportion to the MRR and independent of feed rate of cutting tool and depth of cut. Namely, the MRR has been identified as the main factor for power consumption for material cutting. While in the variable-MRR machining process, the power consumption is in proportion to the MRR and its proportional constant notably depended on the depth of cut and the variation pattern of MRR during the operation. The machining with a shorter interval of MRR changes tended to show a larger power consumption than that of the machining with a longer interval of MRR changes. These facts indicate that the power consumption for metal cutting depends on the tool path and there would be an optimal tool path to minimize the power consumption and tool wear in response to a machining shape.
\end{abstract}

Index Terms-Power consumption, cutting, material removal rate, variation pattern of MRR, tool path.

\section{INTRODUCTION}

Machining represents one of the main energy-consuming activities in manufacturing industries and energy consumption determines $20 \%$ of machine tool operating cost [1]. Reducing the energy consumption by machining process has been identified as the important strategies to improve the tool life as well as the environmental performance [2].

According to the characteristics of material removal rate (MRR), machining process can be grouped into two types; constant-MRR and variable-MRR machining processes. Constant-MRR process is defined as the process that all of the cutting parameter remain unchanged during machining, while variable MRR process is defined as the process that at least one of the cutting parameters change time during machining. The energy consumption characteristics of constant-MRR and variable-MRR machining process are different.

Most of existing studies focused on power or energy modelling of constant-MRR machining process [3]-[9]. The cutting speed, feed rate and depth of cut in constant-MRR machining process are all constant. Hence, the cutting force is

Manuscript received October 16, 2018; revised March 8, 2019. The paper was presented at 2018 3rd International Conference on Design and Manufacturing Engineering (ICDME2018) at Melbourne, Australia

Y. Kondo and Y. Itoh are with Yamagata University, 4-2-16, Yonezawa, Yamagata, 980-8552, Japan (e-mail: kondo@yz.yamagata-u.ac.jp)

Mitsugu Yamaguchi is with Salesian Polytechnic, 4-6-8 Koyamagaoka, Machida, Tokyo, 194-0215, Japan.

S. Sakamoto is with Yokohama National University, 79-2, Tokiwadai, Hodogaya, Yokohama, 240-8501, Japan.

Kenji Yamaguchi is with Yonago National College of Technology, 4448 Hikona, Yoagp, Tottori, 683-0854, Japan a constant value during cutting process. The situation is significantly different, when it comes to variable-MRR process. As the MRR is changing during machining process, cutting force will change with the varying cutting parameters such as the cutting speed, feed rate and depth. Consequently, the power consumption for material cutting will be a dynamic changing value as well. For instance, grooving and end face turning are typical variable-MRR machining processes.

It is easy to be seen that the power consumption of variable-MRR machining process are more complicated than that of constant-MRR machining process. However, up to now, research specially focused on the energy consumption of variable-MRR machining process is really rare [10], [11]. In this study, we tried to examine the power consumption for material cutting in variable-MRR machining process through the plane machining of bakelite sheet with four-depth of grooves.

\section{EXPERIMENTAL PROCEDURE}

\section{A. Measurement of Power Consumption}

The experimental setup for measuring the power consumption for material cutting is shown in Fig. 1. Only electric power consumed by the spindle motor $P_{\text {measure }}$ was measured at 0.2 seconds interval by using a power tester (Hioki model 3333). Then the net power consumption in the cutting process $P_{\text {cut }}$ was estimated by subtract the power consumption at idle running $P_{\text {idle }}$ from the measured spindle power. The evolution of net power consumption is a picture of the evolution of cutting force and a fairly accurate measure of the deterioration of tool condition as shown in equation (1).

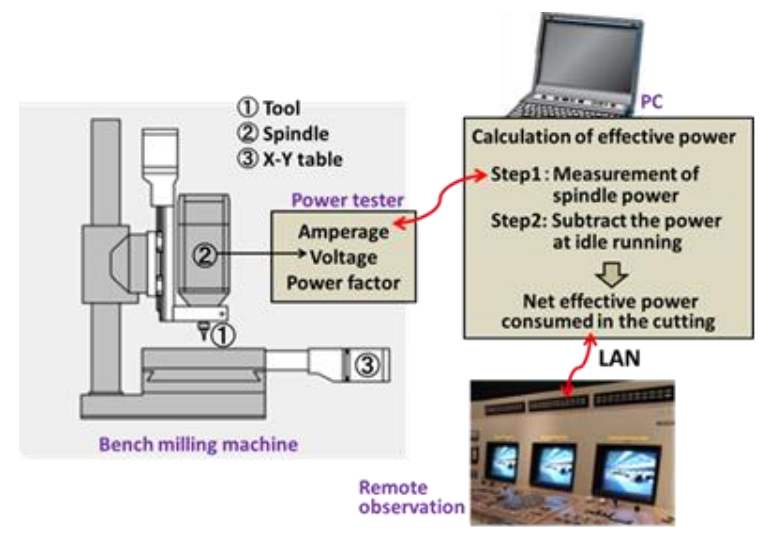

Fig. 1. Experimental set-up for measuring power consumption

$$
P=F_{c} \cdot V /(60 \times 1000 \times \eta)
$$

where, $P[\mathrm{~kW}]$ is net effective power, $F_{\mathrm{c}}[\mathrm{N}]$ is principal 
cutting force, $V[\mathrm{~m} / \mathrm{min}]$ is cutting speed and $\eta$ is mechanical efficiency.

\section{B. Grooving}

A grooving operation was conducted on a vertical bench type CNC machine with an AC spindle motor under the grooving conditions of Table I. The workpiece material used in grooving was a commercial brass plate and the cutting tool was a standard HSS two-flute end mill with a diameter of $4 \mathrm{~mm}$. The power consumption had been measured at 0.2 seconds interval during the machining of grooves with the depth of $0.5,1.0$ and $1.5 \mathrm{~mm}$ at the feed rate of 60,120 and $180 \mathrm{~m} / \mathrm{min}$. In this experiment, the MRR had been kept a constant value during the machining.

\begin{tabular}{l} 
TABLE I: GROOVING CONDITIONS \\
\hline \hline Bench type CNC machine \\
Work: Brass $50 \times 50 \times 5$ \\
Tool : two-flute end mill $\phi 4$ \\
Cutting fluid : none \\
Feed rate : $60 \sim 180 \mathrm{~mm} / \mathrm{min}$ \\
Spindle speed: $7,200 \mathrm{rpm}$ \\
Depth of cut : $0.5,1.0,1.5 \mathrm{~mm}$ \\
Overhanging of tool : $12 \mathrm{~mm}$ \\
\hline \hline
\end{tabular}

\section{Plane Working}

To evaluate the power consumption at variable-MRR process, a plane working of bakelite sheet was conducted. A square bakelite sheet having four grooves with the depth of 1.0, 2.0, 3.0 and 4.0mm were prepared as the workpiece. Then a flat surface was formed by a plane processing using $\Phi 2.0 \mathrm{~mm}$ of HSS two-flute end mill. The cut has a straight shape with the width of $2 \mathrm{~mm}$ and the depth of $1.0,2.0$ or $4.0 \mathrm{~mm}$. The cutting tool had been moved parallel (Y-direction) and vertical (X-direction) to the longitudinal direction of groove to examine the effect of MRR changing condition on power consumption. In the case of moving the tool in Y-direction, the MRR gradually changes at a relatively long interval, while the MRR would vary in a short period when the tool is moved in X-direction as shown in Fig. 2. The depth of cutting is considered as a steady-state load constantly acting on the tool during the machining.

\section{RESULTS AND DISCUSSIONS}

\section{A. Power Consumption at Constant-MRR Process}

Fig. 3 shows the power consumption changes when the grooves with the depth of $0.5,1.0$ and $1.5 \mathrm{~mm}$ were machined at the feed rate of 60,120 and $180 \mathrm{~m} / \mathrm{min}$. In general, the power consumption measured by the wattmeter $P_{\text {measure }}$ is expressed as $P_{\text {idle }}+P_{\text {cut }}$. Where $P_{\text {idle }}$ is the idle power and can be decomposed spindle standby power and spindle rotation power. $P_{\text {cut }}$ is the power consumption to cut the material by cutting edge and is a main factor for dynamic changes of power consumption in the machining. In this study, the power consumption for metal cutting $P_{\text {cut }}$ is defined as the power consumption in the cutting process. The power consumption has been kept an almost constant value during the machining as far as the cutting was conducted under the same condition.
The unit power consumption per second of machining. $P_{\text {unit }}$ is almost in proportion to both the feed rate and the depth of cut, namely $P_{\text {unit }}$ is in proportion to MRR as shown in Fig. 4. This situation can be modelled in Eq. (2).

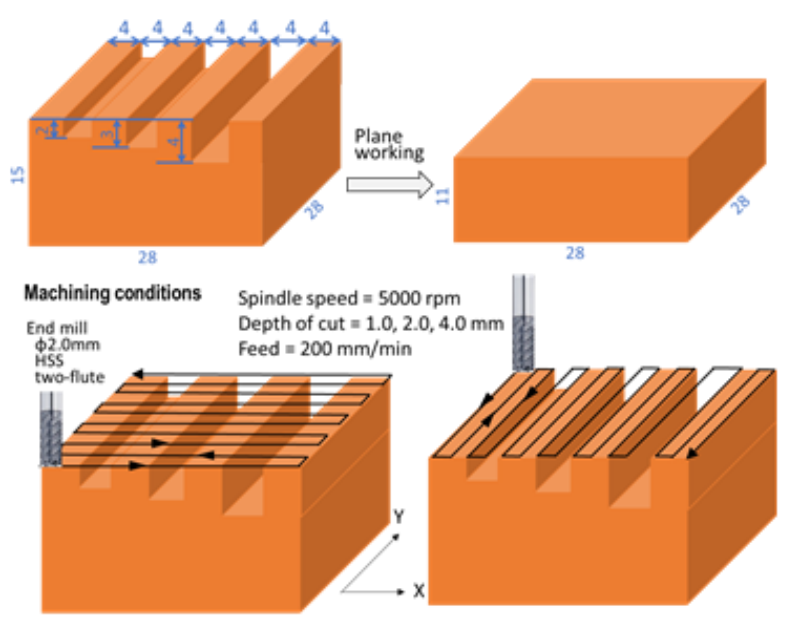

Fig. 2. Summary of plane working.

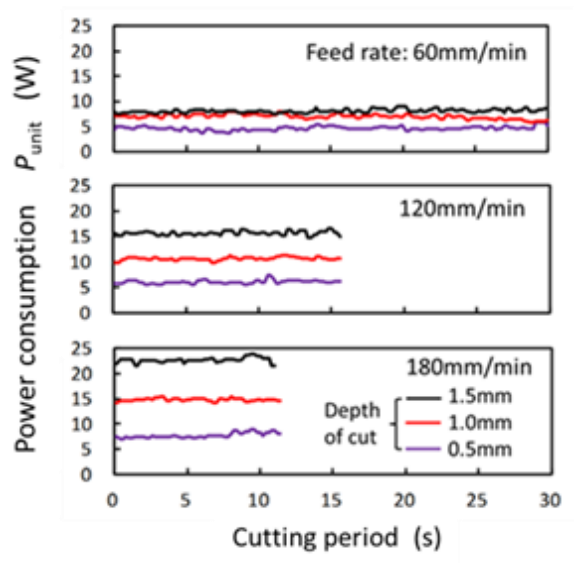

Fig. 3. Power consumption change in grooving.

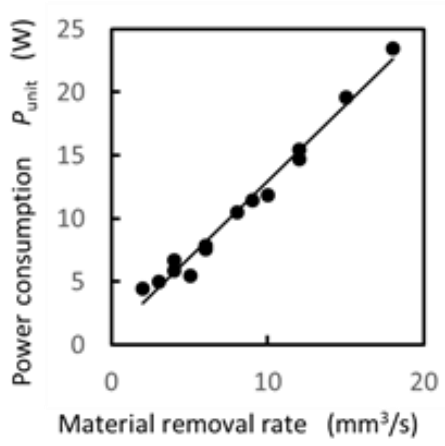

Fig. 4. Relation between power consumption and MRR.

$$
P_{\text {unit }}=k_{0} \cdot M R R
$$

where $k_{0}$ is a constant in $\mathrm{kJ} / \mathrm{mm}^{3}$ and MRR is the material removal rate in $\mathrm{mm}^{3} / \mathrm{s}$. This equation shows that the MRR has been identified as the main factor for power consumption for metal cutting in the constant-MRR machining process.

\section{B. Power Consumption at Variable-MRR Process}

Fig. 5 shows the measured power consumption, $P_{\text {measure, }}$ change when the workpiece was machined in X-direction at $1 \mathrm{~mm}$ of depth of cut. The cutting was conducted three cycles in a law using the same cutting tool. The measured power 
consumption tended to increase with increasing of machining cycle $N_{\mathrm{c}}$. Fig. 6 shows the measured power consumption change when the workpiece was machined in $\mathrm{X}$ and Y-direction at $4 \mathrm{~mm}$ of depth of cut. In the case of the cutting in Y-direction, the measured power consumption had been changed at a relatively long interval, while the cutting in $\mathrm{X}$-direction varied power consumption in a short period as expecting from the MRR changes.

The relation between the depth of cut and the unit power consumption $P_{\text {unit }}$ in the 1 st cycle of machining is summarized in Fig. 7. In the case of variable-MRR process, it is difficult to estimate the material removal rate (MRR) since the MRR has been varied every moment with the cutting time. Therefore, the depth of cut can be considered as the substitute parameter of MRR, because the depth of cut is proportion to MRR theoretically. The unit power consumption is proportion to the depth of cut but showed a difference between the machining in $\mathrm{X}$-direction and $\mathrm{Y}$-direction. The unit power consumption in the cutting of $\mathrm{X}$-direction tended to be enlarged as compared with those of the cutting in Y-direction. These facts mean that the proportional constant $k_{0}$ in equation (2) notably depends on the variation pattern of MRR during the cutting in the variable-MRR process, while $k_{0}$ seems to be a constant value in the constant-MRR process.

\section{MODELLING of Power Consumption for Variable-MRR Process}

Fig. 8 summarizes the total power consumption $P_{\text {total. }}$ the total power consumption $P_{\text {total }}$ is the overall power consumption consumed by the spindle motor over the entire plane working of grooved workpiece. It is expecting that the total power consumption $P_{\text {total }}$ would show the same value unless the tool diameter (groove width), feed rate and rotational speed of tool are invariable. However, the actual $P_{\text {total }}$ was proportional to the depth of cut, in other words $P_{\text {total }}$ was proportional to mean MRR $\left(\mathrm{MRR}_{\text {mean }}\right)$ over the entire plane working and its proportion coefficient $k_{\mathrm{t}}$ depends on the cutting direction and the number of cutting cycle.

Fig. 9 shows the slope $k_{\mathrm{t}}$ and $\mathrm{y}$-interception $P_{\text {base }}$ obtained from the linear regression equation between the depth of cut and total power consumption. The slope and y-interception have a linear relation with the depth of cut. The total power consumption tended to increase with the depth of cut, i.e. mean material removal rate $\mathrm{MRR}_{\text {mean }}$ and its increasing rate depends on the cutting direction and the number of machining cycle $N_{\text {c }}$ i.e. tool wear. Therefore, the total power consumption of variable-MRR machining process can be formulated as follows;

$$
P_{\text {total }}=\left(1+\alpha \cdot N_{c}\right) \cdot k_{t} \cdot M R R_{\text {mean }}+\left(1+\beta \cdot N_{c}\right) \cdot P_{\text {base } 1}
$$

where $P$ base 1 is the y-interception obtained from the linear regression equation in the $1^{\text {st }}$ cycle of machining, $\alpha$ is a correction coefficient depending on the cutting direction and $\beta$ is a correction coefficient depending on the tool wear. $\alpha>0$ and $\beta \fallingdotseq 0$ in the machining with a shorter interval of MRR changes (X-direction), while $\alpha \fallingdotseq 0$ and $\beta>0$ in the case of the machining with a longer interval of MRR changes (Y-direction).

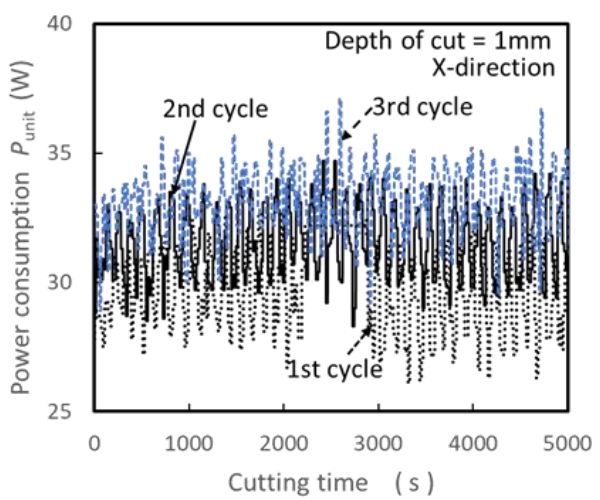

Fig. 5. Power consumption change in plane working.

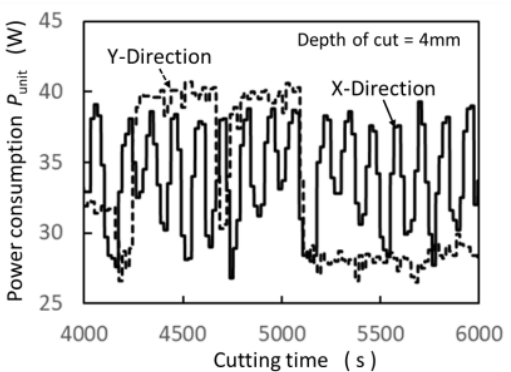

Fig. 6. Relation between power consumption and cutting time.

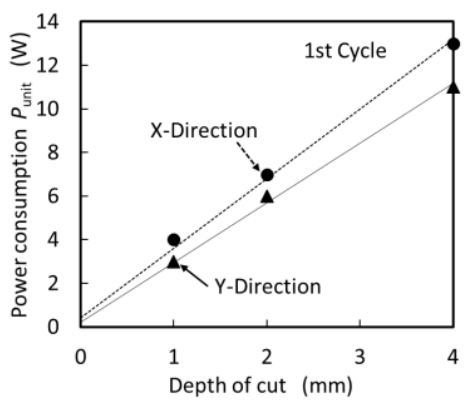

Fig. 7. Relation between depth of cut and power consumption.

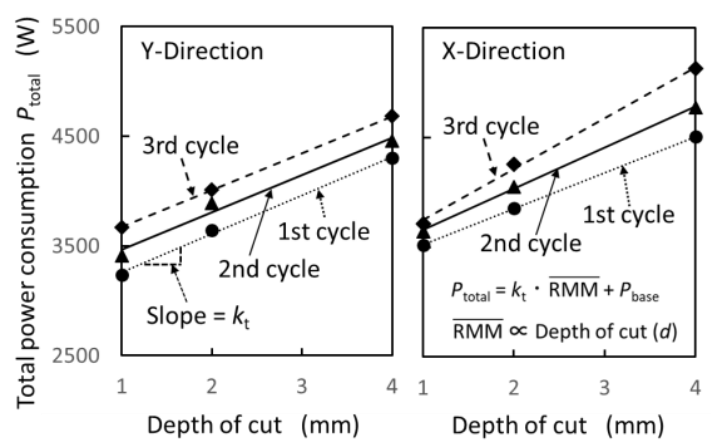

Fig. 8. Total power consumption in plane working.
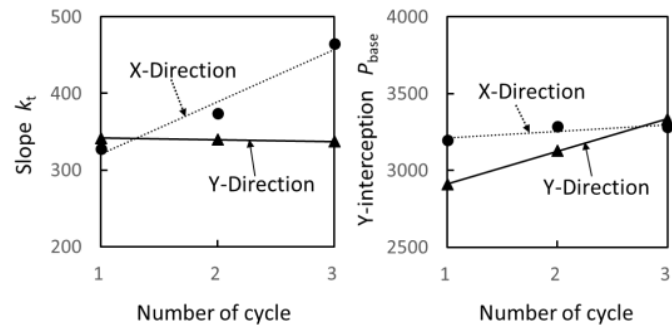

Fig. 9. Slope $k_{\mathrm{t}}$ and y-interception $P_{\text {base. }}$

\section{CONCLUSION}

We tried to examine the power consumption for 
material-cutting in variable-material removal rate (MRR) machining process through the plane machining of bakelite sheet. In the case of constant-MRR process, the power consumption is almost in proportion to the MRR and independent of feed rate of cutting tool and depth of cut. Namely, the MRR has been identified as the main factor for power consumption for material cutting. While in the variable-MRR machining process, the power consumption is in proportion to the MRR and its proportional constant notably depended on the depth of cut and the variation pattern of MRR during the cutting. The machining with a shorter interval of MRR changes tended to show a larger power consumption than that of the machining with a longer interval of MRR changes. These facts indicate that the power consumption for metal cutting depends on the tool path and there would be an optimal tool path to minimize the power consumption and tool wear in response to a machining condition.

\section{REFERENCES}

[1] M. I. Calvanese, P. Alberteli, A. Matta, and M. Taisch, "Analysis of energy consumption in CNC machining cenyers and determination of optimal cutting conditions," in Proc. 20th CIRP Int. Conf. on Life Cycle Engineering, Singapore, 2013, pp. 227-232.

[2] R. K. Bhushan, "Optimazation of cutting parameters for minimizing power consumption and maximizing tool life and empirical modelling," J of Cleaner Production, vol. 39, pp. 242-254, 2013.

[3] S. Rahimifard, Y. Scow, and T. Childs, "Minimizing embodied product energy to support energy efficient manufacturing," CIRP Ann-<anufacturing Technology, pp. 25-28, 2010.

[4] J. X. Lv, S. Jia, R. Z. Tang, "The blig-based energy supply modeling of CNC machine tools," J Clean Prod, vol. 65, pp. 168-177, 2010.

[5] A. Dietmair and A. Verl, "A generic energy consumption model for decision making and energy efficiency optimization in manufacturing," Int J Sustain Eng, vol. 2, no. 2, pp. 123-133, 2009.

[6] A. Dietmair and A. Verl, "Energy consumption forecasting and optimization for tool machines," Modern Machinery Sci., vol. 3, pp. $62-67,2009$

[7] M. Mori, M. Fujishima, Y. Inamasu, and Y. Oda, "A study on energy efficiency improvement for machine tools," CIRP Ann - Manuf Technol, vol. 60, no. 1, pp. 145-148, 2011.

[8] O. Avram and P. Xirouchakis, "Evaluating the use phase energy requirements of a machine tool system," J Clean Production, vol. 19, pp. 699-711, 2011.

[9] O. Avram, I. Stroud, and P. Xirouchakis, "A multi-criteria decision method for sustainability assessment of the use phase of machine tool systems," Int J Adv Manuf Technol, vol. 53, pp. 811-828, 2011.

[10] J. Shun et al., "Energy modelling for variable material removal rare machining process," Int. J of Manuf Technol, vol. 85, pp. 2805-2818, 2016.

[11] L. Lin et al., "Energy requirements evaluation of milling machines based on thermal equilibrium and empirical modeling," J. of Clean Production, vol. 52, pp. 113-121, 2013.
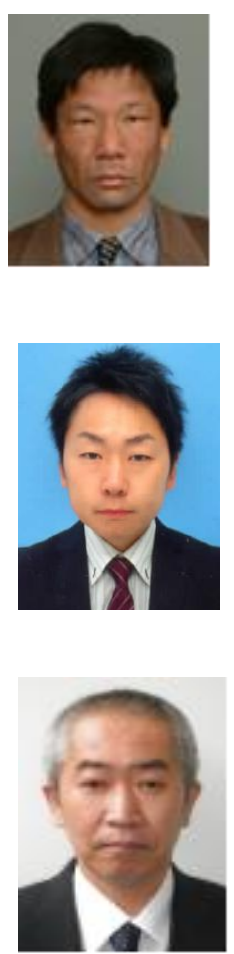

Satoshi Sakamoto received his doctorate from Kanazawa University, Japan in 1997. Now he is a professor of education at Yokohama National University. His current research interests include precision machining of hard and brittle materials. $\mathrm{He}$ is a member of the Japan society for precision engineering, the Japan society of mechanical engineers, and the Japan society for abrasive

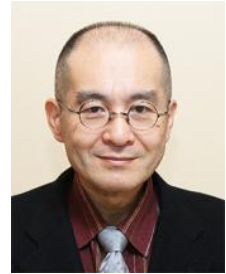

Kenji Yamaguchi received the Ph.D degree in 2002 from Tottori University. His current position is a professor of the Department of Mechanical Engineering, Yonago National College of Technology. His research interests include an effective succession of technicians' skill and a monitoring system for cutting fluids condition. 